\title{
Percutaneous cervical cordotomy for the control of pain in patients with pleural mesothelioma
}

\author{
M B Jackson, D Pounder, C Price, A W Matthews, E Neville
}

\begin{abstract}
Background-Severe chest pain is common in mesothelioma. Percutaneous cervical cordotomy, which interrupts the spinothalamic tract at the $\mathrm{C} 1 / \mathrm{C} 2$ level causing contralateral loss of pain sensation, is particularly appropriate in mesothelioma as the tumour is unilateral and systemic analgesia may be ineffective and is limited by harmful side effects.

Method-A retrospective review was performed to determine the effectiveness and complication rate of this procedure.

Results-Fifty two patients were using opioids prior to cordotomy. The median daily dose of morphine before and after cordotomy was $100 \mathrm{mg}$ (range $\mathbf{0 - 1 0 0 0} \mathrm{mg}$ ) and $20 \mathrm{mg}$ (range $\mathbf{0 - 5 2 0} \mathrm{mg}$ ), respectively $(p<0.001)$. Forty three patients $(83 \%)$ had a reduction in pain such that their dose of opioid could be at least halved. Twenty patients $(38 \%)$ were able to stop completely. Recurrence of pain requiring an increase in opioid medication was recorded in 18 patients at a median time of nine weeks (range 0.7-26 weeks). Four patients developed mild weakness, two had troublesome dysaesthesia. The median time from cordotomy to death was 13 weeks (range 0.3-52 weeks). Six early deaths within two weeks of cordotomy occurred early in the series and reflect postoperative chest infection and poor selection as the patients were in the terminal stages of mesothelioma.
\end{abstract}

Department of Respiratory Medicine M B Jackson

A W Matthews

E Neville

Department of Pain

Control

D Pounder

C Price

Portsmouth Hospitals NHS Trust,

Portsmouth, UK

Correspondence to: Dr M B Jackson,

Department of Respiratory

Medicine, Southampton

General Hospital,

Southampton SO16 6YD,

UK.

Received 16 June 1998

Returned to author

17 August 1998

Revised manuscript received

26 October 1998

Accepted for publication

17 November 1998 from mesothelial cells which occurs most commonly in the pleura. In most cases there is a history of exposure to asbestos which is causally related. ${ }^{1}$ The incidence of malignant mesothelioma is still increasing and it is predicted that it will continue to do so into the early part of the next century. ${ }^{2}$ It is a common condition in those geographical areas where there is a large population of dockyard workers who have been subjected to heavy asbestos exposure in the past. Severe intractable chest pain, known as the costopleural syndrome, ${ }^{34}$ is a common feature of pleural mesothelioma as the tumour often invades the pleural cavity and thoracic wall early in the disease process. In a survey of 200 patients pain was a presenting symptom in $58 \% .^{5}$ Systemic analgesia is generally the first choice of treatment to relieve most cases of cancer pain; however, nociceptive pathway block should be considered early in the costopleural syndrome as systemic analgesia often fails to give sufficient relief. ${ }^{34}$

Surgical section of the anterolateral tract of the spinal cord in the treatment of persistent pain was described at the beginning of this century. ${ }^{6}$ The technique has since been refined using electrode thermocoagulation. Percutaneous cervical cordotomy interrupts the spinothalamic tract at $\mathrm{C} 1 / 2$ by means of a controlled radiofrequency electric current, causing contralateral loss of pain sensation below the level of the lesion. ${ }^{78}$ Complete lesion of the spinothalamic tract can render virtually one half of the body pain free. The technique of percutaneous cervical cordotomy is well described elsewhere. ${ }^{9}$

Percutaneous cervical cordotomy is well recognised for the treatment of the costopleural syndrome, ${ }^{31011}$ and may be particularly appropriate when this is caused by pleural mesothelioma. There is usually unilateral disease, but it is too diffuse to allow other more local nociceptive pathway blocks to be effective.

In this retrospective review we describe the use of percutaneous cervical cordotomy in the control of pain in 53 patients with malignant pleural mesothelioma, studying the effectiveness and complication rate of the procedure. Patients with pleural mesothelioma were selected for percutaneous cervical cordotomy when they developed pain due to chest wall invasion. Inevitably this meant that the patient had started opioid medication, usually in combination with a non-steroidal antiinflammatory drug, as simple analgesia alone had by this stage proved ineffective in obtaining pain control. After effective cordotomy the dose of opioid is halved to prevent respiratory depression. Further reductions can be made more slowly over the ensuing two weeks to prevent withdrawal symptoms.

\section{Methods}

A retrospective review was performed of all available notes of patients who had undergone cordotomy for pain from pleural mesothelioma since 1985 . Where data collection was incomplete, information was sought by letter to the patient's general practitioner. To increase objectivity the information was obtained and 
evaluated by one of us (MBJ) who was not involved with the work of the Pain Clinic.

\section{Results}

The records of 53 patients (52 men) were obtained. Data on opioid dose were available at least until two weeks after the procedure (or until death if this occurred sooner) in 52 patients $(98 \%)$. Information was requested from the patient's general practitioner in five cases and the date of death was subsequently obtained in four instances. The median age at the time of cordotomy was 64 years (range 44-82). The median time from the diagnosis of pleural mesothelioma to cordotomy was 21 weeks (range three days to 143 weeks). The median time from diagnosis to death in 51 patients was 24 weeks (range 10 days to 164 weeks). At the time of presentation 40 patients $(75 \%)$ had a pleural effusion, eight $(15 \%)$ had a pleural mass/thickening, and one patient had pleural encasement by tumour. The type of pleural involvement was not clear from the case notes in the remaining four patients $(8 \%)$.

PAIN RELIEF

Forty eight of the 53 patients (91\%) were taking controlled release morphine sulphate tablets, often in conjunction with morphine elixir as required. Seven patients (13\%) were using a diamorphine infusion. Only one patient was not taking either of these drugs at the time of cordotomy, as they had caused troublesome side effects previously. There is no direct equation defining the relationship between equivalent doses of oral morphine and subcutaneous diamorphine but, for the purposes of this analysis, we have used an equivalence used commonly in palliative care. ${ }^{12}$

Thirty six of the 53 patients $(68 \%)$ had taken or were taking co-analgesic medication in addition to morphine/diamorphine. The most frequently used were non-steroidal, antiinflammatory drugs, which had been taken by 31 patients $(58 \%)$.

After percutaneous cervical cordotomy 20 patients $(38 \%)$ were able to stop taking morphine/diamorphine completely. A further $19(37 \%)$ continued on regular opioid medication but at less than half of the dose prior to the procedure. Overall, in 43 of 52 patients $(83 \%)$ there was an improvement in pain, allowing a $50 \%$ or greater reduction in opioid dose.

The median daily dose of morphine before cordotomy was $100 \mathrm{mg}$ (range $0-1000 \mathrm{mg}$ ) and the median lowest daily dose of morphine after cordotomy was $20 \mathrm{mg}$ (range 0-520 mg), $\mathrm{p}<0.001$ (Mann-Whitney test). After percutaneous cervical cordotomy, co-analgesic medication was continued in 18 patients (34\%), eight of whom were managed solely on a nonsteroidal anti-inflammatory drug and 10 on a combination of non-steroidal antiinflammatory drug with either morphine or carbamazepine. Twenty three patients (43\%) were not taking co-analgesic medication after cordotomy. This had been discontinued in seven patients after a successful procedure, and in the other patients had been considered unhelpful prior to the procedure. In the remaining 12 patients it was not clear from the case records whether co-analgesic medication had been prescribed after percutaneous cervical cordotomy.

Four of the 53 patients (8\%) proceeded to a second cordotomy because of initial failure in producing adequate relief, and this was successful in three instances.

Recurrence of severe pain requiring a subsequent increase of opioid was noted in 18 patients $(34 \%)$. The median time for this recurrence in the 14 case notes in which it was recorded was nine weeks (range five days to six months).

\section{COMPLICATIONS}

Two patients experienced troublesome dysaesthesia following cervical cordotomy and persistent motor weakness was noted in four. This was only graded in one patient (4/5, Medical Research Council scale for grading muscle function), although in the other three it was not regarded as severe. No patient suffered hemiplegia or was unable to walk as a result of their weakness. Unfortunately no data were found on the duration of this weakness in any patient.

The median time from cordotomy to death in 51 patients was 13 weeks (range two days to 52 weeks). Three patients $(6 \%)$ died within one week of cordotomy and there were three other early deaths within two weeks of the procedure. In terms of pain control, the procedure had been a success in five of these patients and none had needed a second procedure to achieve a satisfactory result. Two of these patients were severely disabled by dyspnoea at rest because of pleural encasement from their tumours, and the third patient had marked cachexia and a very short life expectancy due to the mesothelioma itself. Two of the other patients who died early developed evidence of a chest infection following cordotomy, with pyrexia and a cough productive of green sputum. No information was available regarding the sequence of events leading to death in the sixth patient.

There were no other complications recorded. In particular, no patient developed incontinence due to sphincter disturbance. Impotence and postural hypotension can be expected in bilateral cordotomy (which this unit does not practise because of the higher incidence of central sleep apnoea, the "Ondine syndrome"1314), but are usually rare in unilateral cordotomy and were not recorded.

\section{Discussion}

It can be inferred that most of the patients in this series $(83 \%)$ had a significant reduction in pain, and 20 of $52(38 \%)$ were able to stop opioid medication completely. This is an important finding in pleural mesothelioma as the pain is often severe and opioid resistant. This is a retrospective review, however, with all the problems inherent therein. Some data were difficult to confirm because we can only be sure of relevant data that were recorded in the individual case notes. In particular, the lowest dose of morphine following the procedure, the use 
of co-analgesic medication, the recurrence of pain, and the incidence of minor complications may not have been recorded in some cases. In any procedure where the primary aim is palliation, quality of life should be considered, and it can be argued that the dose of opioid is not necessarily the best indicator of a satisfactory result in cordotomy. In a retrospective review, though, it is the best quantifiable variable. In a prospective study other data could have been recorded such as visual analogue pain scales and quality of life assessment with validated questionnaires. It would be reasonable to assume, however, that a treatment producing effective control of previously severe chronic pain, without the detrimental effect of significant side effects, would also lead to a substantial improvement in quality of life. In this series most of the patients who had a documented reduction in opiate dose also had additional positive comments recorded such as "complete relief", " $100 \%$ better", "pain free", etc, but in the context of this retrospective review these cannot be quantified satisfactorily.

The alternatives to percutaneous cervical cordotomy are not as useful. High doses of opioid in those patients whose pain is partially responsive are associated with significant side effects which may themselves shorten life expectancy. Co-analgesic medication such as non-steroidal anti-inflammatory drugs and drugs affecting pain fibre conduction such as carbamazepine and amitriptyline can be used to treat mesothelioma pain, but in our experience are not very effective. Other techniques to interrupt pain fibres can also be used-for example, intercostal nerve block and intrathecal block-however, most of the patients in this series presented with disease that diffusely affected the hemithorax, making these treatments inappropriate. Palliative radiotherapy was not used in this series for pain control for the same reason. It is our practice, however, to use radiotherapy prophylactically at pleural intubation sites to prevent fistulation of the pleural tumour. ${ }^{15}$ One patient in this series underwent limited pleurectomy before referral for cordotomy but none of the other patients in this series had treatment that was any more than palliative in the treatment of their pleural malignancy. The published success rate for percutaneous cervical cordotomy in the control of pain from both benign and malignant causes is $76-89 \% . .^{916-18}$ Unsuccessful cordotomy results from the anatomical variations encountered.

Eighteen patients (34\%) went on to develop a recurrence of severe pain requiring a subsequent increase in opioid dose, but this was often after a useful period of good pain control. The recurrence of pain was due to further spread into the mediastinum or peritoneum.

There have been a number of staging classifications described for pleural mesothelioma, although none is definitive. All the patients in this series had pain secondary to chest wall invasion, putting them into stage 2 or above of the commonly used classification by Butchart, ${ }^{19}$ or stage 4 of a recently proposed system based on the TNM classification. ${ }^{20}$ Staging was performed with the aid of information from plain chest radiographs in all patients, thoracoscopy in 32 patients (60\%), and computed tomographic (CT) scans of the thorax in 19 patients $(36 \%)$. The latter investigation, however, should not be regarded as a definitive assessment in these circumstances as it is known to give a significant rate of false negatives in detecting chest wall invasion in this disease. ${ }^{212}$ For those patients early in the series, before CT scanning became part of the routine management of pleural mesothelioma, and for those patients where CT scanning could not confirm chest wall invasion, the diagnosis was made at the onset of severe intractable pain typical of chest wall involvement, bearing in mind the natural history of this disease. It is important, however, not to confuse this pain with the dragging discomfort of bulky tumour confined to the parietal pleura without chest wall invasion.

This study has highlighted the low rate of complications that we have observed with this procedure. The incidence of troublesome dysaesthesia was similar to previous studies ${ }^{78} 16$ and there was no case of severe motor weakness or sphincter disturbance. Thermoanaesthesia frequently occurs following cervical cordotomy, and all patients are warned about this and advised appropriately. Horner's syndrome

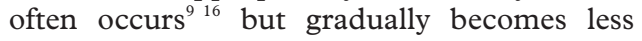
obvious and is not troublesome.

The published mortality at one week for percutaneous cervical cordotomy carried out for all causes of persistent pain (both benign and malignant) is $1-6 \% .^{9}$ When only those with malignant disease are considered, the reported mortality at one week is $6 \% .{ }^{16}$ Considering that all of the patients in this series had mesothelioma, some with advanced disease, our results are comparable.

The dose of morphine, time from diagnosis to procedure, and age of the patients who died early within two weeks of the procedure (median $120 \mathrm{mg}, 26$ weeks, and 58 years, respectively) did not differ significantly from the remainder of the patients in the series. However, this group of patients did have advanced disease and were probably less able to tolerate percutaneous cervical cordotomy as a result, as the procedure may worsen respiratory failure in susceptible individuals or in those on opioids by causing respiratory depression. In the upper cervical region there are ascending and descending respiratory reticular fibres ${ }^{131423}$ close to the region where the upper cervical nociceptive fibres lie and, if a high level of analgesia is required (as in pleural mesothelioma), then these fibres may be damaged. If the patient has little reserve in the unaffected lung then a unilateral cordotomy, carried out contralateral to the pain, may temporarily reduce respiration to a life threatening degree. ${ }^{24}$ This matter is the subject of an ongoing study in this unit. With tighter patient selection the early mortality in this series could be improved, however, and these results support the use of percutaneous cervical cordotomy for the control of otherwise intractable pain which often 
occurs at or soon after presentation in malignant mesothelioma, before the patient enters the terminal stages of their illness. It is the practice of this unit to offer cordotomy to patients when pain due to chest wall involvement, the "costopleural syndrome", is first suspected.

In conclusion, high percutaneous cervical cordotomy is successful in controlling the pain from malignant pleural mesothelioma as assessed by a reduction in opioid dose. There is a low rate of significant complications in this series. Percutaneous cervical cordotomy should be considered in patients with pleural mesothelioma as soon as pain due to chest wall invasion is suspected.

Conflict of interest: none

1 Wagner JC, Skeggs CA, Marchand P. Diffuse pleural mesothelioma and asbestos exposure in the North Western Cape Province. Br F Ind Med 1960;17:260-71.

2 Peto J, Hodgson JT, Matthews FE, et al. Continuing increase in mesothelioma mortality in Britain. Lancet 1995;345 535-9.

3 Watson PN, Evans RJ. Intractable pain with lung cancer. Pain 1987;29:163-73.

4 Bonica JJ. Chest pain related to cancer. In: JJ Bonica, ed. The of pain. Philadelphia: Lea and Febiger, 1990:1083-113.

5 Matthews AW. A survey of malignant mesothelioma in Portsmouth 1982-1991. Thorax 1992;47:851-2.

6 Spiller WG, Martin E. The treatment of persistent pain of organic origin in the lower part of the body by division of organic origin in the lower part of the body by division of the anterolateral

7 Mullan S, Hekmetpanam J, Dobbin G, et al. Percutaneous intramedullary cordotomy utilising the unipolar anoda electrolytic lesion. $\mathcal{F}$ Neurosurg 1965;22:548-53.

8 Rosomoff HL, Carroll F, Brown J, et al. Percutaneous radiofrequency cervical cordotomy technique. $\mathcal{F}$ Neurosurg 1965;23:639-44.

9 Lipton S. Percutaneous cordotomy. In Wall PD, Melzack R, eds. Textbook of pain. Edinburgh: Churchill Livingstone, 1989: 832-9.
10 Ischia S, Ischia A, Luzzani A, et al. Results up to death in the treatment of persistent cervico-thoracic (Pancoast) and thoracic malignant pain by unilateral percutaneous cervical cordotomy. Pain 1985;21:339-55.

11 Schrottner O. Results of percutaneous cordotomy in lung and breast cancer: a comparative study with strong support for a multidimensional nature of pain. Pain Clin 1991;4: $217-22$

12 Prescribing in palliative care. British National Formulary 1997;34:12-15.

13 Hitchock E, Leece B. Somatotopic representation of the respiratory pathways in the cervical cord of man. $f$ Neurosurg 1967;27:320-9.

14 Krieger AJ, Rosomoff HL Sleep induced apnoea. Part 1. A respiratory and autonomic dysfunction syndrome following bilateral percutaneous cervical cordotomy. $f$ Neurosurg bilateral percutan

15 Lowe E, Khoury GG, Matthews AW, et al. Prevention of tumour seedlings following thoracoscopy in mesothelioma by prophylactic radiotherapy. Clin Oncol 1995;7:317-8.

16 Lahuerta J, Bowsher D, Lipton S, et al. Percutaneous cervical cordotomy: a review of 181 operations on 146 patients cal cordotomy: a review of 181 operations on 146 patients with a study on location of "pain fibres" in the C-2

17 Nagaro T, Amakawa K, Yamauchi Y, et al. Percutaneous cervical cordotomy and subarachnoid phenol block in pain control of costopleural syndrome. Pain 1994;58:323-30.

18 Sanders M, Zuurmond W. Safety of unilateral and bilateral percutaneous cervical cordotomy in 80 terminally ill cancer patients. F Clin Oncol 1995;13:1509-12.

19 Butchart EG, Ashcroft T, Barnsley WC, et al. Pleuropneumonectomy in the management of diffuse malignant mesothelioma of the pleura: experience in 29 patients. Tho$\operatorname{rax}$ 1976;31:15-24.

20 Rusch VW, Venkatraman E. The importance of surgical staging in the treatment of malignant pleural mesothelioma. F Thorac Cardiovasc Surg 1996;111:815-26.

21 Maasilkta P, Vehmas T, Kirasaari L, et al. Correlation between findings at computed tomography (CT) and at thoracoscopy/thoracotomy/autopsy in pleural mesothelioma. Eur Respir f 1991;4:952-4.

22 Rusch VW, Godwin JD, Shuman WP. The role of computed tomography in the initial assessment and the follow up of malignant pleural mesothelioma. F Thorac Cardiovasc Surg 1988;96:171-7.

23 Henderson VE, Craigie EH. On the respiratory centre. $A m$ f Physiol 1936;115:520-9.

24 Mullan S, Hosobuchi Y. Respiratory hazards of high cervical percutaneous cordotomy. 7 Neurosurg 1968;22:531-8. 